Mots. Les langages du politique

\title{
Les élections municipales au miroir de la presse quotidienne régionale. Des cadres médiatiques aux thématiques politiques
}

The municipal elections through the mirror of the regional daily press. From media frameworks to political thematics

Las elecciones municipales dentro de la mirada de la prensa quotidiana regional. Desde los marcos mediáticos a las temáticas políticas

\section{Emmanuel Marty}

\section{OpenEdition}

Journals

Édition électronique

URL : https://journals.openedition.org/mots/21991

DOI : 10.4000/mots.21991

ISSN : 1960-6001

Éditeur

ENS Éditions

Édition imprimée

Date de publication : 6 octobre 2015

Pagination : 39-55

ISBN : 978-2-84788-727-3

ISSN : 0243-6450

\section{Référence électronique}

Emmanuel Marty, « Les élections municipales au miroir de la presse quotidienne régionale. Des cadres médiatiques aux thématiques politiques », Mots. Les langages du politique [En ligne], 108 | 2015, mis en ligne le 06 octobre 2017, consulté le 23 avril 2022. URL : http://journals.openedition.org/mots/ 21991 ; DOI : https://doi.org/10.4000/mots.21991 


\section{Les élections municipales au miroir de la presse quotidienne régionale. Des cadres médiatiques aux thématiques politiques}

La question des thématiques politiques mises en avant dans les médias dits d'information politique et générale se pose de manière particulièrement saillante en période électorale. Notre objectif ici est d'interroger la manière dont la presse quotidienne régionale ${ }^{1}$ concourt à privilégier ou au contraire à minorer, dans l'espace public, certains enjeux et facettes des élections. Les municipales de mars 2014 constituent une période propice à l'analyse du traitement opéré par la $P Q R$, au sein d'un discours dans lequel se superposent les figures du lecteur et de l'électeur comme récepteurs des productions journalistiques. La PQR représente en effet, comme le rappelle opportunément Franck Bousquet (2014), 80\% des quotidiens vendus en 2013. Sa situation, marquée par le monopole de la plupart des titres sur leurs zones respectives de diffusion, devra tout d'abord être exposée, pour tenter de comprendre comment peut se forger dans ses colonnes une certaine image des territoires. Ce sera l'objet de la première partie du texte.

Les théories du cadrage médiatique développées notamment par Shanto lyengar (1991), William Gamson (1992) et Robert Entman (1993) dans les champs conjoints des sciences politiques et de la psychologie sociocognitive, seront convoquées comme outils conceptuels opératoires pour l'analyse du corpus. Nous nous attacherons dans une deuxième partie à préciser en quoi leur mobilisation en amont de l'usage d'une statistique lexicale cooccurrentielle ouvre des perspectives pour l'appréhension de la médiatisation du politique en période électorale. Ces théories nous paraissent d'autant plus appropriées que Shanto lyengar et Donald Kinder (1987) ont déjà montré comment le processus de cadrage médiatique pouvait chez le récepteur, par un phénomène d'amorçage, influencer profondément les critères jugés pertinents pour évaluer les acteurs politiques et les thématiques qu’ils privilégient ${ }^{2}$.

1. Désormais: $P Q R$.

2. Bien que l'étude de référence d'lyengar et Kinder concerne plus directement la télévision, l'ensemble des travaux nord-américains sur les cadres médiatiques sont parvenus à des conclusions similaires concernant la presse écrite.

Université Nice Sophia Antipolis, Information Milieux Médias Médiations (13M) emmanuel.marty@unice.fr 
La statistique lexicale cooccurrentielle, plus précisément la classification descendante hiérarchique de Max Reinert (1983), sera mobilisée dans une troisième partie pour outiller l'analyse d'un corpus d'articles de PQR pendant la campagne. Ce corpus, balayant transversalement le territoire français, doit nous permettre de faire émerger les espaces conceptuels rendus saillants dans la médiatisation des élections municipales. Notre ambition, sur le plan méthodologique, est en effet de présenter la manière dont différentes analyses lexicométriques, s'appuyant sur la théorie des cadres et mobilisant la cooccurrence comme principal outil d'analyse, peuvent nous aider à identifier et caractériser ces espaces conceptuels, dont certains peuvent être liés à des normes journalistiques, et d'autres relever de thématiques politiques émergeant sur les différents territoires. In fine, il s'agit d'approcher les différentes questions politiques mises en visibilité dans le discours de la $P Q R$, par un parcours d'analyse renseigné par les indicateurs statistiques, mais nécessitant un retour au texte pour en affiner l’interprétation.

\section{La presse quotidienne régionale, lieu de l'expression politique d'un territoire?}

La presse a pour fonction communément admise de concourir à l'alimentation du débat démocratique, en jouant pour les citoyens un rôle d'outil d'appréhension de la vie sociale. La PQR occupe dans ce contexte une place particulière, en ce qu'elle constitue le lieu de cristallisation des expressions politiques locales dans des territoires relativement bien circonscrits, qui sont ceux de leurs lectorats. C'est d'autant plus vrai que la plupart des titres se trouvent dans une situation de monopole sur leurs territoires de diffusion. Bernard Lamizet (2013) définit le territoire comme une «médiation politique de l'espace», précisant qu'il «désigne les modalités selon lesquelles les identités politiques se manifestent dans l'espace sous la forme de l'emprise des pouvoirs et de l'étendue de l'expression de la citoyenneté» (p.41-42). Si la dernière formule concerne directement l'alimentation du débat politique, «l'emprise des pouvoirs» désigne pour sa part très explicitement le fait que tout territoire se définit également par l'identité des institutions qui le gouvernent. Or la PQR dépend largement du paysage économique et politique local : comme le montre Bousquet (2014), les institutions politiques locales et, dans une moindre mesure, les grandes entreprises d'un territoire ont la plupart du temps sur celle-ci un poids économique important, historique et structurel. Elles cumulent en effet les statuts de sources, d'annonceurs privilégiés et d'abonnés des titres. Ce poids leur confère un rôle indéniable de prescripteur de l'information locale, qu'il s'agit de ne pas négliger dans l'appréhension du discours de la PQR en période électorale. 


\title{
Des cadres médiatiques aux thématiques politiques : parcours théorique et méthodologique
}

\author{
Les cadres médiatiques : un concept opératoire pour l'analyse \\ du discours journalistique
}

En tant que production de sens par le discours, l'activité journalistique se manifeste par certains actes énonciatifs caractéristiques, tels que rapporter, décrire, commenter ou encore mettre en scène. Jean-Pierre Esquenazi (2002) avance que « le travail de journaliste ne fait que généraliser une activité cognitive familière et [...] les moyens qu'il emploie s'inspirent de ceux qui facilitent à l'homme la compréhension de son propre milieu.» (p.15). C'est précisément sur cette activité de mise en sens de l'expérience par le langage que se sont penchés les théoriciens du cadrage médiatique. Outre-Atlantique, l'étude des cadres médiatiques (media frames) s'est constituée en courant de recherche depuis les années quatre-vingt, à la suite des travaux fondateurs de Todd Gitlin (1980). L'auteur souhaitait interroger les processus psycho-socio-langagiers par lesquels les médias procèdent à une construction de sens dirigée vers leurs publics. Les travaux de Gitlin font bien sûr référence à ceux de Erving Goffman (1991), pour qui les cadres sont des schémas d'interprétation destinés à structurer de manière intelligible et cohérente l'ensemble des stimuli auxquels nous sommes exposés. En les appliquant aux médias, Gitlin les conçoit comme des processus tacites qui contribuent à organiser le monde à la fois pour les journalistes et pour leurs publics. Ainsi les journalistes appliquent-ils dans leur traitement de l'actualité un éventail limité de cadres, qui restreint le nombre d'alternatives dont disposent les publics lorsqu'ils reconstruisent en réception la réalité sociale (Reese, 2007). Ces cadres fonctionnent donc comme des outils conceptuels auxquels médias et individus se fient pour transmettre, interpréter et évaluer l'information, en vertu d'une culture et d'une expérience supposées communes, mises en jeu dans le contrat de communication de l'information médiatique (Charaudeau, 1997).

Dès lors, la couverture des élections municipales peut proposer une mise en avant variable de différentes thématiques entre un titre et l'autre, un territoire et l'autre, en fonction de la nature des débats y ayant cours voire des champs d'action privilégiés par leurs prescripteurs informationnels respectifs. Gamson (1992) considère les cadres comme étroitement liés aux routines journalistiques et au crédit qu'un journaliste attribue à ses différentes sources. Erik Neveu (2009), dans une relecture des travaux des auteurs clés des media frames, évoque une compétition des cadrages impliquant la capacité, inégale, de collectifs aux intérêts hétérogènes sinon divergents à se constituer en sources du discours journalistique. La tension entre pluralité de l'expression 
politique et prédominance des positions du pouvoir institué, présente dans la définition du territoire donnée par Lamizet (2013), doit alors se manifester par certaines modalités discursives. En privilégiant certains aspects de la réalité, le processus de cadrage impliquerait en effet d'en délaisser d'autres, dans un fonctionnement analogue à celui de l'agenda-setting3.

\section{Les cadres médiatiques et la cooccurrence lexicale}

Dans un travail de référence, Entman (1993) conçoit le cadrage comme le fait de définir une situation, d'en déterminer les acteurs ou les causes, puis d'émettre éventuellement des jugements de valeur. William Gamson et André Modigliani (1989) avaient auparavant proposé une définition largement concordante en considérant que les cadres associaient des éléments tels que métaphores, exemples, slogans, descriptions et images, mais convoquaient également dans l'organisation du discours des relations de causes et de conséquences, ou encore l'expression de valeurs morales. Les cadres médiatiques seraient donc constitués non seulement de relations paradigmatiques, mais également de syntagmes traduisant des intentions, stratégies ou routines discursives de l'énonciateur. Les dimensions opérationnelles de leur identification sont débattues depuis de nombreuses années, mais la méthode consistant à regrouper des segments d'énoncés en grappes (ou clusters) en fonction de cooccurrences lexicales est aujourd'hui largement utilisée et considérée par de nombreux auteurs comme fiable et permettant de travailler sur des corpus de taille importante (Matthes, Kohring, 2008 ; Tankard, 2001).

Si l'enthousiasme du champ américain des media frames pour ces méthodologies est légitime, ces dernières doivent toutefois être questionnées sur leur capacité à identifier, par la prise en compte de formes lexicales cooccurrentes, de véritables espaces conceptuels du discours. Pour Pierre Lafon (1984), les cooccurrences recouvrent potentiellement plusieurs réalités textuelles : «syntagmes cassés par l'indexation, stéréotypes, accords thématiques, affinités verbales marquées, habitudes stylistiques, etc.» (p.136). Nous souscrivons alors à l'affirmation de Damon Mayaffre (2014) selon laquelle «considérer la cooccurrence, c'est allier l'urne et le réseau, le paradigmatique et le syntagmatique » (p. 29), dans la mesure où la cooccurrence porte à la fois une dimension statistique et une dimension «co(n)textuelle » en préservant, au moins en partie, la séquence textuelle. La cooccurrence multiple semble ainsi particulièrement adaptée à l'identification des cadres médiatiques dans le langage, par le repérage et le regroupement d'éléments dont les liens revêtent une fonction sémantique. Dans un récent travail, des analyses lexicométriques croisées avec

3. Les media frames sont à ce titre parfois considérés, notamment par Maxwell McCombs (2005), comme un agenda-setting de second niveau. 
une approche plus ontologique du langage (Ledouble et al., 2014) ont en effet apporté une nouvelle illustration de la manière dont les ressources sémantiques de la langue fonctionnaient comme des potentialités de construction de sens, mobilisées, agencées et structurées syntaxiquement en situation de communication, donnant corps à des messages qui renferment de nécessaires cohérences sémiotiques, lesquelles peuvent être reconstruites par l'identification de «mondes lexicaux» (Reinert, 2008).

Pour Reinert, en effet, qui est à l'origine de la classification hiérarchique descendante4, la cooccurrence multiple serait mieux à même de saisir le sens du discours que ne le serait une approche strictement ontologique du langage. Ces considérations font écho à la philosophie du langage de Mikhaïl Bakhtine (1977), pour qui « seul le courant électrique de la communication verbale fournit au mot la lumière de sa signification » (p.147). Reinert (2008), pour sa part, affirme que «le contenu n'est pas dans le mot; il est dans l'acte, dont le mot est une trace» (p. 983).

Ce que les outils lexicométriques quantifient et regroupent, ce sont donc des traces lexicales, c'est-à-dire des marques d'intentions de construction de sens laissées par différents énonciateurs. Aussi, passer de l’identification des traces à la reconstruction du sens implique non seulement un retour au texte dans le processus d'interprétation, pour mettre les indices statistiques au service d'une meilleure intelligibilité des énoncés, mais également l'appréhension du cadre communicationnel des discours, lequel éclaire le sens de l'acte d'énonciation. Nous nous associons ainsi à Bénédicte Pincemin (2012) pour qui le travail interprétatif vise, à partir du regroupement statistique de «cooccurrents au plan des signifiants», à obtenir des «corrélats au plan des signifiés ».

\section{Classification hiérarchique descendante et séquences textuelles}

C'est par le concept opératoire de cadre médiatique que nous souhaitons procéder à ce travail interprétatif. Pour identifier les cadres rendus saillants dans notre corpus, nous utilisons la classification descendante hiérarchique développée par Reinert (1983) et implémentée dans le logiciel Iramuteq5 (Ratinaud, Déjean, 2009). Cette classification repose sur un découpage des textes en segments de plus ou moins 40 occurrences $^{6}$, puis sur la construction d'un tableau lexical à double entrée croisant ces segments avec l'absence ou la présence

4. Voir infra pour une présentation de la méthode.

5. Interface de R pour les analyses multidimensionnelles de textes et de questionnaires. Développé sous licence GNU/GPL par Pierre Ratinaud, Lerass, Université Toulouse 3, http://www. iramuteq.org/.

6. C'est-à-dire que le corpus est découpé en segments de 40 mots dans l'ordre linéaire du texte. Cette unité de 40 occurrences est celle choisie par défaut dans le logiciel Iramuteq, modifiable et toujours modérée par la présence de caractères de ponctuation pour éviter de briser arbitrairement syntagmes ou propositions. 
des formes pleines qui les composent7, préalablement lemmatisées. C'est sur la base de ce tableau binaire que la classification proprement dite est réalisée, les classes lexicales ${ }^{8}$ étant alors issues du regroupement des formes significativement cooccurrentes à l'intérieur de la matrice formée par les segments. Il s’agit donc de regrouper des traces lexicales, caractérisées par une cooccurrence multiple. Pour Nikos Kalampalikis (2003):

Une redondance de traces lexicales circonscrit un lieu usuel du discours, un lieu donc où un certain vocabulaire est utilisé fréquemment, un lieu référentiel où le sujet-énonciateur semble avoir déposé du sens. La méthode Alceste permet donc de mettre en lumière les traces lexicales les plus prégnantes de ces espaces de référence, les «mondes lexicaux». (p.150)

Il s'agit donc bien de passer des cooccurrents aux corrélats, Kalampalikis considérant que la méthode de classification descendante hiérarchique permet l'identification d'un réseau de significations et non uniquement de signifiants. Cette classification, initialement conçue pour l'analyse des représentations sociales, a précédemment été mise en œuvre à plusieurs reprises pourl'analyse du cadrage médiatique de l'identité nationale (Marty et al., 2013) ou de sondages précédant l'élection présidentielle de 2012 (Touboul et al., 2012). À l'issue des classifications, plusieurs options permettent un retour au texte, par des outils s'attachant à en repérer des séquences de tailles et de statuts différents. Le premier type de séquence correspond aux segments de texte dits caractéristiques, dont le repérage est implémenté dans le logiciel Iramuteq. Cette fonctionnalité permet d'extraire, parmi les segments ayant constitué le matériau de la classification hiérarchique descendante (CHD) ceux considérés comme étant les plus caractéristiques de chacune des classes. Sur le plan statistique, ces segments sont affichés par ordre décroissant du total des $\mathrm{KHI} 2$ respectifs des formes marquées du segment. Le second type de séquence communément utilisé est celui des segments répétés. Leur identification après une CHD permet de repérer les expressions figées (Lafon, Salem, 1983) emblématiques de chacune des classes lexicales.

\section{Constitution du corpus}

Douze titres de $P Q R 9$ balayant le territoire français ont constitué les sources de notre corpus de presse, dans leur version papier. À partir de la base de don-

7. Par défaut, Iramuteq considère comme formes pleines les substantifs, verbes, adjectifs, adverbes et formes dites «non reconnues », c'est-à-dire absentes du dictionnaire de lemmatisation, correspondant souvent à des noms de personnes ou de lieux.

8. Pour une description plus statistique des procédés, voir Reinert (1983), ou Ratinaud et Marchand (2012) pour le fonctionnement spécifique à Iramuteq.

9. La Provence, Midi Libre, La Dépêche du Midi, Sud-Ouest, La Charente Libre, Ouest France, La Voix du Nord, L'Est Républicain, Le Parisien, Le Progrès de Lyon, La Montagne, La Nouvelle République 
nées Factiva ont été collectés, en texte intégral, tous les articles comportant le mot-clé «municipales», au pluriel, recouvrant implicitement le syntagme "élections municipales», lors des huit premiers jours de la période officielle de campagne, soit du lundi 10 mars au lundi 17 mars 2014. Pour nous assurer de la validité et de la cohérence du corpus, un nettoyage a été effectué par un parcours attentif de celui-ci. Ont ainsi été écartés des articles contenant la forme «municipales» mais n'abordant pas la question des élections (traitant de rencontres sportives ou culturelles mentionnant les «installations municipales ») ${ }^{10}$, ou jugés non pertinents (agendas dans lesquels le jour du vote pour les municipales figurait parmi un grand nombre d'événements du territoire, brèves annonces indiquant exclusivement les lieux et dates de réunions publiques de candidats, etc.). En revanche les comptes-rendus de ces réunions ont été conservés, de même que les présentations de listes et candidats. Le corpus a enfin été codé pour Iramuteq ${ }^{11}$ afin d'identifier la source et la date de chaque article. Ainsi nettoyé et codé pour le logiciel, notre corpus est constitué de 7045 articles, 2016763 occurrences et 55784 formes lexicales.

\section{La PQR en campagne, des cadres aux thèmes}

La classification descendante hiérarchique effectuée sur le corpus a découpé celui-ci en 57942 segments de 40 occurrences en moyenne, dont 55777 (soit $96,26 \%$ ont contribué à constituer une classe lexicale. En tout, neuf classes lexicales peuvent être distinguées (voir tableau 1, page suivante).

Sur les différentes classes issues de cette analyse, certaines découlent de cadrages médiatiques routiniers, tandis que d'autres, relevant de cadrages différents, contiennent l'expression éventuellement programmatique de thématiques politiques. À la lumière de la théorie des media frames, plusieurs regroupements interprétatifs peuvent être faits entre certaines classes, afin de faire émerger des cadrages distincts construits par les titres de presse. Le retour à la séquence textuelle à l'intérieur des classes lexicales, par l'extraction de segments caractéristiques ou l’identification de segments répétés, permettra d'affiner et d'illustrer nos interprétations.

du Centre-Ouest. Certains titres, tels que Nice Matin, L'Union ou Les Dernières Nouvelles d'Alsace, ne proposaient sur Factiva que leur édition en ligne, très différente de leur version papier. Ils n'ont pas été intégrés au corpus pour en conserver l'homogénéité des supports et contenus.

10. On notera d'ailleurs comment la cooccurrence simple peut ici désambiguïser et sémantiser l'adjectif «municipal».

11. Ce travail d'extraction et de codage du corpus a été effectué avec l'aide précieuse de Pierre Ratinaud. Qu'il en soit chaleureusement remercié. 


\section{Tableau 1. Récapitulatif des profils lexicaux des classes issues de la classification hiérarchique descendante}

\begin{tabular}{|c|c|c|}
\hline $\begin{array}{l}\text { Classes et } \\
\text { pourcentage } \\
\text { du corpus }\end{array}$ & $\begin{array}{c}\text { Profil lexical } \\
\text { (20 formes lemmatisées par Khi2 décroissant) }\end{array}$ & $\begin{array}{l}\text { Journaux liés à la classe } \\
\text { (par Khi2 décroissant) }\end{array}$ \\
\hline $\begin{array}{l}\text { Classe } 1 \\
17,27 \%\end{array}$ & $\begin{array}{c}\text { campagne, voir, gens, aller, chose, parler, connaître, } \\
\text { page, politique, prendre, passer, semaine, temps, } \\
\text { jour, moment, question, sourire, monde, taubira, } \\
\text { facebook }\end{array}$ & $\begin{array}{l}\text { La Voix du Nord } \\
\text { Le Parisien } \\
\text { La Provence } \\
\text { La Charente Libre }\end{array}$ \\
\hline $\begin{array}{l}\text { Classe } 2 \\
14,78 \%\end{array}$ & $\begin{array}{c}\text { liste, municipal, élection, présenter, maire, mandat, } \\
\text { prochain, tête, candidat, conduire, sortir, ensemble, } \\
\text { briguer, représenter, conseiller, décider, élire, } \\
\text { suffrage, seul, lice }\end{array}$ & $\begin{array}{l}\text { La Montagne } \\
\text { Ouest France } \\
\text { Le Progrès } \\
\text { La Nouvelle République }\end{array}$ \\
\hline $\begin{array}{c}\text { Classe } 3 \\
7,42 \%\end{array}$ & $\begin{array}{c}\text { électoral, vote, document, lettre, carte, droit, } \\
\text { distribuer, livret, bulletin, courrier, panneau, tract, } \\
\text { envoyer, boîte, recevoir, foi, propagande, pli, citoyen, } \\
\text { signer }\end{array}$ & $\begin{array}{l}\text { Le Parisien } \\
\text { La Voix du Nord } \\
\text { L’Est Républicain } \\
\text { La Charente Libre } \\
\text { La Provence }\end{array}$ \\
\hline $\begin{array}{l}\text { Classe } 4 \\
16,75 \%\end{array}$ & $\begin{array}{l}\text { création, aménagement, zone, logement, mettre, } \\
\text { place, construction, rénovation, scolaire, plan, } \\
\text { centre-ville, créer, bâtiment, projet, parking, eau, } \\
\text { stationnement, voirie, espace, réseau }\end{array}$ & $\begin{array}{l}\text { Ouest France } \\
\text { Sud-Ouest } \\
\text { Le Progrès }\end{array}$ \\
\hline $\begin{array}{l}\text { Classe } 5 \\
12,72 \%\end{array}$ & $\begin{array}{l}\text { vie, commune, développement, associatif, action, } \\
\text { économique, village, compétence, souhaiter, qualité, } \\
\text { projet, lien, service, équipe, population, habitant, } \\
\text { écouter, social, développer, communauté }\end{array}$ & $\begin{array}{l}\text { Le Progrès } \\
\text { Ouest France } \\
\text { La Dépêche du Midi }\end{array}$ \\
\hline $\begin{array}{c}\text { Classe } 6 \\
9,91 \%\end{array}$ & $\begin{array}{l}\text { gauche, ump, ps, front, droite, socialiste, fn, marine, } \\
\text { national, tour, pen, udi, partir, sondage, législatif, } \\
\text { modem, bleu, score, extrême, communiste }\end{array}$ & $\begin{array}{l}\text { Le Parisien } \\
\text { La Provence } \\
\text { Midi Libre }\end{array}$ \\
\hline $\begin{array}{c}\text { Classe } 7 \\
5,79 \%\end{array}$ & $\begin{array}{l}\text { impôt, euro, taux, baisser, taxe, budget, } \\
\text { investissement, dépense, augmenter, } \\
\text { fonctionnement, recette, augmentation, dette, } \\
\text { fiscal, imposition, emprunt, dotations, habitation, } \\
\text { endettement, montant }\end{array}$ & $\begin{array}{l}\text { Sud-Ouest } \\
\text { Ouest France }\end{array}$ \\
\hline $\begin{array}{c}\text { Classe } 8 \\
5,87 \%\end{array}$ & $\begin{array}{c}\text { h, salle, mars, réunion, organiser, vendredi, heure, } \\
\text { mercredi, jeudi, public, mardi, fête, samedi, } \\
\text { lundi, dimanche, } 18 \text { h3o, polyvalent, rendez-vous, } \\
\text { renseignement, rue }\end{array}$ & $\begin{array}{l}\text { Midi Libre } \\
\text { Sud-Ouest } \\
\text { La Dépêche du Midi } \\
\text { La Voix du Nord } \\
\text { La Charente Libre }\end{array}$ \\
\hline $\begin{array}{c}\text { Classe } 9 \\
9,49 \%\end{array}$ & $\begin{array}{c}\text { retraité, agriculteur, agent, conseiller, assistant, } \\
\text { technicien, marier, employé, jean, sortir, professeur, } \\
\text { infirmier, artisan, compatble, secrétaire, commercial, } \\
\text { chauffeur, agricole, adjoint, responsable }\end{array}$ & $\begin{array}{l}\text { La Montagne } \\
\text { La Nouvelle République } \\
\text { L’Est Républicain } \\
\text { La Dépêche du Midi } \\
\text { Le Progrès } \\
\text { Midi Libre } \\
\text { La Charente Libre }\end{array}$ \\
\hline
\end{tabular}




\section{L'information institutionnelle et la non-médiation journalistique}

Les classes 2, 8 et 9 peuvent être regroupées, en ce qu'elles constituent les modalités d'une information d'ordre essentiellement institutionnel, peu ou pas filtrée par une médiation journalistique, comme nous allons le voir. La classe 2 , tout d'abord, regroupe les énoncés s'attachant strictement à annoncer les candidats aux élections et le nom de leur liste, avec une forte présence des candidatures à une réélection, comme en témoignent les formes maire, prochain, mandat, représenter et sortir (forme réduite de sortant) ${ }^{12}$. La classe 8 , dans le sillage de la précédente, est constituée par les annonces de réunions publiques organisées par les différents candidats, tandis que la classe 9 est structurée par les compositions détaillées des listes, énonçant quasi systématiquement les nom, état civil, âge et profession des candidats ${ }^{13}$.

La quasi-totalité des titres de PQR apparaissent dans au moins l'une de ces trois classes ${ }^{14}$, accréditant l'idée d'un cadrage généralisé dans la presse locale. Ces classes peuvent être considérées comme relevant de «l'information-service » telle que définie par Bousquet (2014), c'est-à-dire « un type d'information factuelle, issue d'une source la légitimant sans qu'aucune instance éditoriale n'intervienne sur le fond de son contenu et donnant à son récepteur des éléments lui permettant d'agir dans des domaines économiques, sociaux ou culturels» (p. 21). L'absence manifeste d'intervention éditoriale de la part des journalistes et la dimension utilitaire de l'information pour le lecteur sont deux éléments constitutifs de cette «information-service», dont l'adéquation à ces trois classes lexicales doit être explicitée.

La présentation des candidats, des noms et slogans de listes, leur composition détaillée ou encore l'annonce des réunions publiques organisées constituent dans notre corpus des contenus dont les véritables énonciateurs ne sont pas les journalistes, comme c'est d'ordinaire la règle dans l'information journalistique, marquée par une distance entre l'énonciateur et l'objet référentiel du discours (Mouillaud, Tétu, 1989), mais bien les candidats euxmêmes. C'est la raison pour laquelle on peut considérer ces trois classes lexicales comme relevant d'une non-médiation journalistique, en ce sens que l'activité de cadrage des énoncés, censément prise en charge par les journalistes,

12. Sur 5494 occurrences de la forme réduite sortir, 5123 correspondent en effet à la forme initiale sortant, recouvrant le syntagme maire sortant.

13. Les brèves annonces de réunions publiques ont été exclues du corpus du fait de leur pauvreté informationnelle. Les annonces dont il est question ici font partie d'articles plus vastes et constituent la classe 8. Les listes de la classe 9 ont pour leur part été laissées dans le corpus car jugées comme faisant partie intégrante de l'information électorale délivrée par la PQR. Le fait que ces deux types spécifiques d'information aient été extraits pour constituer chacun une classe lexicale semble accréditer la validité de la CHD pour notre corpus.

14. Àl'exception du Parisien et de La Provence, dont certaines spécificités sont abordées plus bas. 
est ici entièrement déléguée aux sources, dès lors à la fois émetteurs et objets de l'intégralité de ces messages. Bousquet (2014) considère d'ailleurs cette information de nature institutionnelle et non éditorialisée comme constitutive de la PQR. Héritière à la fois de l'annonce et de l'information administrative, parfois accusée d'être au service des institutions locales, elle conserve néanmoins une dimension utilitaire importante pour le lecteur, notamment en zone rurale où elle demeure un relai privilégié d'informations pratiques sur les élections, précisément par la présentation des candidats et des listes, par l'annonce des dates et lieux de réunions publiques.

\section{Médiation routinière et normalisée : le cadrage médiatique épisodique}

La classe 1, marquée par des verbes d'action et des déclaratifs, consiste essentiellement en un récit des campagnes de terrain menées par les différents candidats. Elle se distingue assez nettement des précédentes : il ne s'agit pas ici d'information-service telle que précédemment définie, dans la mesure où les journalistes sont bien, dans le cas présent, les véritables énonciateurs des discours. Ces récits s'apparentent plutôt à de l'information de proximité, narrant la plongée des candidats dans l'univers des administrés d'un territoire, leur rencontre dans des lieux symboliques de campagne tels que les marchés ou les pas-de-porte de riverains. Les articles qui relèvent de cette classe lexicale sont ponctués par le discours des candidats, invités par les journalistes à livrer leur sentiment sur ces rencontres et dont les paroles sont rapportées et intégrées au récit journalistique. Le passage suivant est caractéristique ${ }^{15}$ de cette classe : « «Les gens sont contents de nous voir, on discute longtemps avec eux, reprend Jean-François Castell. Des gens qui ne votaient plus nous disent qu'ils vont y retourner. " Le candidat aura encore intensifié le rythme pendant la campagne officielle» (La Voix du Nord, 14 mars). La classe 3 se situe également dans un récit de la campagne, mais regroupe plutôt les discours relatant les démarches, procédures et matériels de vote, tels que la réception des cartes d'électeurs et des professions de foi par les citoyens, les panneaux électoraux, le dépôt des listes en préfecture ou encore les procurations. Y sont parfois relatées les cérémonies organisées par certains maires pour remettre aux nouveaux votants un carnet d'électeur, comme dans le passage caractéristique suivant: "40 jeunes de Vayres sont devenus majeurs dans l'année et ils ont été conviés à la mairie samedi pour recevoir des mains d'Hélène Maidon leur carte électorale et le livret citoyen présentant les principes fondamentaux de la démocratie.» (Sud-Ouest, 13 mars)

La classe 6 relève quant à elle d'une routine journalistique bien connue de la recherche sur le discours médiatique en période électorale. Articulés autour

15. Au sens statistique du terme, voir supra. 
des résultats de sondages, des stratégies et alliances des candidats et partis politiques, ces récits relèvent de ce qu’lyengar (1991) a nommé le cadrage "course de chevaux» (Horse race framing), focalisé sur la compétition électorale et la rivalité de ses protagonistes, y compris dans une dimension prospective destinée à entretenir un certain suspense quant à l'issue de cette compétition. Depuis les années quatre-vingt-dix, plusieurs auteurs (Champagne, 1994 ; Blondiaux, 1998) pointent la place grandissante accordée aux sondages dans la presse, poussant les journalistes à déléguer aux experts leur autorité et leur responsabilité d'explication des réalités sociales. Très fréquent dans la presse quotidienne nationale, ce type de cadrage étroitement lié à la communication politique reste présent dans la PQR. Les titres faisant le plus fort usage de ce cadrage sont d'ailleurs Le Parisien et La Provence, titres des deux premières villes de France dont les protagonistes ont une forte dimension nationale, ainsi que Midi Libre, couvrant une région marquée par la présence de candidats très médiatiques du Front national. Les formes Aliot, Ménard et Collard appartiennent en effet spécifiquement à cette classe de discours. Or il a déjà été montré dans un précédent travail (Touboul et al., 2012), à travers l'exemple de Marine Le Pen, que les candidats du Front national suscitaient de manière accrue ce cadrage médiatique, du fait d'un certain renoncement journalistique au débat politique, souvent risqué à la fois pour le journal et le journaliste, au profit du discours d'expertise objectivé et légitimé par les sondages.

Ce cadrage "course de chevaux» est une déclinaison de ce qu'lyengar (1991) a défini comme un «cadrage épisodique», dans lequel les deux classes précédentes s’inscrivent pleinement. Pour lyengar, en effet, le cadrage épisodique est un cadrage court, du point de vue tant spatio-temporel que conceptuel, souvent individualisant, dont la visée est d'illustrer un sujet par des cas précis, des exemples concrets, se déroulant dans l'immédiateté. Les portraits de candidats en campagne et les récits de leurs rencontres avec les citoyens, dans les classes 1 et 3, s'inscrivent bien dans ce type de cadrage. L'information de proximité vise d'abord à faire connaître et à valoriser les différents candidats en mettant en scène leur travail de terrain dans l'espace local. Roselyne Ringoot et Yvon Rochard (2005) ont déjà montré comment journalistes et politiques ont érigé la proximité en loi. Les récits de terrain lisses et déconflictualisés, dont les journalistes sont les auteurs, et les candidats à la fois les objets et les sources privilégiées, en sont l'expression emblématique, dont on peut penser qu'il s'agit d'une trace discursive de l'emprise structurelle des pouvoirs sur la PQR. Iyengar distingue ce cadrage épisodique d'un autre type de cadrage, dit " thématique », dans lequel les médias s'attachent à élargir la focale pour aborder véritablement les éléments de fond d'un sujet. Un tel cadrage serait susceptible de contenir les principales thématiques abordées et débattues dans les campagnes électorales, car ces dernières seraient explicitement présentées et posées dans le discours de la PQR comme objets politiques. 


\section{Projets, programmes et thématiques politiques}

C'est dans les classes 4, 5 et 7 que vont véritablement transparaître des questions ou projets politiques mis en saillance dans la période électorale. Y apparaissent des substantifs et des courts syntagmes, identifiés comme segments répétés, que l'on peut raisonnablement considérer comme des thématiques politiques, parfois convoquées par les candidats dans leurs projets. Ainsi la classe 4 renferme-t-elle un certain nombre de points programmatiques des candidats, mis en avant dans les colonnes de la PQR comme des enjeux politiques saillants dans l'espace local. On y trouve en premier lieu les questions d'urbanisme, avec le plan local d'urbanisme ou PLU, mais aussi l'aménagement, la création, la construction ou encore la rénovation de divers espaces, bâtiments ou équipements municipaux. Le logement, le transport, les questions de circulation et de stationnement sont très présentes, notamment en ce qui concerne le centre-ville. On trouve également dans cette classe de nombreuses mentions de la mise en place des nouveaux rythmes scolaires, votés nationalement mais dont l'application incombe aux municipalités, ou encore la question de la gestion de l'eau. Les discours de cette classe puisent largement dans les professions de foi des équipes, à l'image de ce passage : «Les projets futurs sont la construction d'un local pour l'accueil périscolaire et la cantine à proximité de l'école mais aussi la création de logements dans l'ancien restaurant du Campagnard, l'aménagement des rues, des travaux de voirie et l'avenir du bâtiment de l'Adapei. " (Le Progrès, 14 mars) Ainsi cette classe renvoiet-elle à certaines des compétences essentielles des municipalités, telles que l'urbanisme, la voirie et l'enseignement. La gestion des installations, équipements et services publics de la commune apparaît alors dans les colonnes de la PQR comme des enjeux électoraux dont se saisissent les candidats en les intégrant dans leurs projets, comme en témoignent le verbe créer et le syntagme verbal mettre en place. À travers la présentation d'éléments programmatiques, relativement précis, mis en avant par les différents candidats aux élections, les journalistes de PQR semblent ici désireux de satisfaire le besoin d'information des citoyens vis-à-vis des offres politiques locales.

La classe 5 se structure quant à elle autour d'un discours porté par l'intention et la finalité du projet politique, plus que par ses manifestations matérielles. On y voit apparaître ce qui s'apparenterait à des champs de l'action politique, plus larges que les projets eux-mêmes, tels que le développement économique, le développement durable, l'emploi, le tourisme, l'action sociale, la vie associative, le sport et la culture. Sont également présentes des notions ne relevant pas à proprement parler de champs de compétence mais plutôt de véritables thématiques politiques mises en avant par les candidats, telles que le lien social, la qualité de vie, la solidarité, la jeunesse ou encore la démocra- 
tie participative. Cette dimension à la fois plus conceptuelle et idéologique des discours est marquée par les verbes souhaiter, écouter, développer, améliorer ou encore préserver, marquant l'engagement politique des candidats dans leurs discours, comme dans le passage suivant : «la formation, qui aligne dixneuf candidats, s'est fixé des objectifs prioritaires : l'action sociale et la qualité de vie, le maintien et le développement de l'économie (agriculture, artisanat, commerce) ou la préservation du patrimoine et de l'environnement» (Ouest-France, 12 mars).

La classe 7, quant à elle, est relativement spécifique. Elle relève du bilan de gestion des communes livré par les candidats sortants et restitué par les journalistes. Ces bilans sont le plus souvent focalisés sur l'impôt et les taxes, mentionnant la dette et détaillant le budget dans ses recettes et ses dépenses de fonctionnement et d'investissement. Les propositions avancées par les équipes sortantes pour le prochain budget sont alors relayées, avec une nécessaire distance, dans les colonnes de la PQR. Elles portent essentiellement sur le niveau des contributions financières demandées ainsi que sur la faisabilité économique de certains projets, à l'image du passage suivant : "La liste se dit soucieuse de ne pas augmenter les taux des impôts locaux. Les deux opérations ont donc été conçues pour que les recettes, ou les économies, compensent les dépenses.» (Sud-Ouest, 12 mars).

Il se développe ainsi au sein de ces trois classes une information balayant les projets et thématiques politiques mis en avant par les candidats à l'élection qui, bien que largement fondée sur leurs supports de communication électorale, fait néanmoins l'objet d'une véritable médiation journalistique par laquelle la $P Q R$ joue son rôle d'éclairage des citoyens dans leurs choix électoraux. Les projets, exposés par les candidats et mis en discussion par les journalistes, sont ceux de l'espace local, liés aux compétences particulières des municipalités. Leur teneur est donc sensiblement différente de celle des débats ayant cours à l'échelle nationale, souvent plus marqués idéologiquement. Peu de journaux, finalement, se situent significativement dans ce cadrage thématique : Sud-Ouest, Ouest France, Le Progrès et La Dépêche du Midi. On pourrait en déduire que les territoires sur lesquels sont diffusés ces titres font preuve d'une plus grande ouverture politique, par la place laissée à la présentation et la discussion de différents projets, sans toutefois pouvoir juger à ce stade dans quelle mesure cette discussion serait l'expression d'une diversité politique en termes idéologiques.

\section{Vers une cartographie lexicale des thématiques politiques}

À la suite de l'identification des cadrages précédemment réalisée, il devient envisageable de cartographier de manière plus systématique les thématiques 
politiques soulevées dans les différents territoires. Cette identification peut alors s'appuyer sur une nouvelle CHD, effectuée sur chacune des trois classes relevant du cadrage thématique, soit les classes 4, 5 et 7. À titre d'exemple méthodologique, une CHD sur les énoncés de la classe 5 permet de diviser celle-ci en plusieurs classes, chacune délimitant à la fois des thématiques mises en saillance, des journaux particuliers et des espaces locaux relativement précis. Àl'intérieur de ces sous-classes, en effet, des noms de communes, candidats ou forces politiques récurrents peuvent aisément être identifiés ${ }^{16}$. L'une de ces classes, par exemple, propre à Ouest France et concernant au premier chef la commune de Machecoul (Loire Atlantique), est structurée par la concertation et la démocratie participative. Une autre se focalise sur la question du lien intergénérationnel et se révèle particulièrement associée aux communes de Plouay (Morbihan) et de Nieppe (Nord), de manière saillante dans les titres de PQR diffusés dans ces deux territoires, soit respectivement OuestFrance et La Voix du Nord. Une autre encore, utilisée par Midi Libre et associée d'abord au PS, est formée par le développement économique associé à la préservation de l'environnement et du patrimoine. Les résultats peuvent, de la sorte, être analysés de manière plus systématique et la focale de l'analyse encore resserrée ${ }^{17}$ pour faire émerger des liens entre thématiques et territoires, acteurs politiques ou médiatiques particuliers, plus difficilement repérables de prime abord ${ }^{18}$.

Par un parcours interprétatif de notre corpus d'articles, fondé sur le concept opératoire de cadre médiatique et outillé par le repérage des cooccurrences lexicales, différents types de médiations journalistiques opérées par la PQR en période électorale auront pu être mis au jour : la non-médiation journalistique, qui correspond à une information de service de nature quasi administrative, une information de proximité, passant par un cadrage épisodique plutôt personnifiant, dépolitisé et étroitement dépendant de la communication politique, et un cadrage thématique correspondant à une réelle médiation journalistique s'attachant à mettre en visibilité dans l'espace public local des champs et projets politiques, devenant de ce fait des enjeux électoraux pour les candidats comme pour les (é)lecteurs. Si les deux premiers types de cadrage semblent explicitement relever de l'emprise des pouvoirs locaux sur la PQR, le dernier

16. Le repérage est facilité par la possibilité de classer les formes lexicales des profils de classes selon leur catégorie, l'ensemble des noms de communes, candidats et partis se trouvant dans la catégorie «nr», non reconnus.

17. Les résultats de cette dernière étape sont donnés à titre illustratif, comme piste d'approfondissement de l'analyse des thématiques politiques. L'exposé détaillé et systématique des résultats de cette nouvelle analyse constitue nous semble-t-il un autre travail, qui dépasserait l'enjeu méthodologique de la présente contribution.

18. Des outils tels que les analyses de similitudes, proposées par le logiciel Iramuteq et centrées sur la visualisation des cooccurrences lexicales, peuvent alors être mobilisés pour obtenir une représentation graphique globale de ces connexions. 
renferme en revanche «l'expression de la citoyenneté » évoquée par Lamizet (2013). L'analyse détaillée des classes relevant du cadrage thématique peut alors permettre, par un effort d'analyse systématique des indicateurs statistiques, d'esquisser une cartographie des thèmes et acteurs rendus saillants dans la médiatisation par les titres de PQR de la campagne des élections municipales. Si les outils de la statistique lexicale cooccurrentielle facilitent considérablement le repérage des traces de l'activité discursive des locuteurs, seul un travail interprétatif outillé théoriquement et nourri par le retour au texte permet le passage des cooccurrents aux corrélats et l'identification effective des thématiques politiques.

\section{Références}

BAKHTINE Mikhaïl, 1977, Le marxisme et la philosophie du langage. Essai d'application de la méthode sociologique en linguistique, Paris, Minuit.

BLondiaux Loïc, 1998, La fabrique de l'opinion. Une histoire sociale des sondages, Paris, Le Seuil.

BOUSQUET Franck, 2014, Pour une approche globale de l'information infranationale. Éléments d'analyse du papier au numérique, mémoire d'HDR, Université Toulouse 3.

Champagne Patrick, 1994, "De la doxa à l'orthodoxie politologique», Actes de la recherche en sciences sociales, $\mathrm{n}^{0}$ 101-102, mars 1994, p. $23-24$.

Charaudeau Patrick, 1997, Le discours d'information médiatique. La construction du miroir social, Paris, Nathan, INA.

ENTMAN Robert, 1993, «Framing. Toward clarification of a fractured paradigm », Journal of Communication, $\mathrm{n}^{\circ} 43$ (4), p. 51-58.

ESquenAZI Jean-Pierre, 2002, L'écriture de l'actualité. Pour une sociologie du discours médiatique, Presses universitaires de Grenoble.

Gamson William, 1992, Talking Politics, Cambridge, Cambridge University Press.

Gamson William, ModiglianI André, 1989, "Media discourse and public opinion on nuclear power. A constructionist approach», American Journal of Sociology, n०95 (1), p. 1-38.

GITLIN Todd, 1980, The Whole World Is Watching, Berkeley, Los Angeles, University of California Press.

GoffMAn Erving, 1991, Les cadres de l'expérience, Paris, Minuit.

IYENGAR Shanto, 1991, Is Anyone Responsible? How Television Frames Political Issues, Chicago, The University of Chicago Press.

IYENGAR Shanto, KINDER Donald, 1987, News that Matters. Television and American Opinion, Chicago, The University of Chicago Press.

KALAMPALIKIS Nikos, 2003, "L'apport de la méthode Alceste dans l'analyse des représentations sociales", Méthodes d'étude des représentations sociales, J.-C. Abric éd., Paris, Erès, p. 147-163. 
LAFON Pierre, 1984, Dépouillements et statistiques en lexicométrie, Genève-Paris, Slatkine-Champion.

LAFON Pierre, SALEM André, 1983, "L'inventaire des segments répétés d'un texte», Mots. Les langages du politique, n 6, L'œuvre de Robert-Léon Wagner. Vocabulaire et idéologie. Analyses automatiques, p. 161-177.

LAMIZET Bernard, 2013, «Mutations de la citoyenneté », E-citoyennetés, A. Krasteva éd., Paris, L'Harmattan, p.41-58.

Ledouble Hélène, Marty Emmanuel, CADel Peggy, 2014, "Sémantique et statistique lexicale. Approches croisées de l'analyse de discours pour l'accompagnement du changement organisationnel», Les discours organisationnels sous toutes leurs formes. Récits, discours, conversations, interactions, 82e colloque de l'ACFAS, Université Concordia, Montréal, Canada, mai 2014.

Marty Emmanuel, Marchand Pascal, Ratinaud Pierre, 2013, "Les médias et l'opinion. Éléments théoriques et méthodologiques pour une analyse du débat sur l'identité nationale», Bulletin de méthodologie sociologique / Bulletin of Sociological Methodology, no 117 (1), p. 46-60.

MATTHES Jörg, KoHRING Matthias, 2008, «The content analysis of media frames. Toward improving reliability and validity ", Journal of Communication, n 58 , p. 258-279.

MAYAFFre Damon, 2014, «Plaidoyer en faveur de l'analyse de données co(n)textuelles. Parcours cooccurrentiels dans le discours présidentiel français (1958-2014) », JADT $2014: 12^{e}$ Journées internationales d'analyse statistique des données textuelles, Paris.

McComBs Maxwell, 2005, «A look at agenda-setting. Past, present and future », Journalism Studies, nº 6, p. 543-557.

MouIlLAud Maurice, TÉTu Jean-François, 1989, Le journal quotidien, Lyon, Presses universitaires de Lyon.

Neveu Erik, 2009, Sociologie du journalisme, Paris, La Découverte.

PINCEMIN Bénédicte, 2012, «Sémantique interprétative et textométrie», Texto! Textes et cultures, $\mathrm{n}^{\circ} 17$ (3), p. 1-21.

Ratinaud Pierre, DÉjean Sébastien, 2009, «Iramuteq. Implémentation de la méthode Alceste d'analyse de texte dans un logiciel libre», Modélisation appliquée aux sciences humaines et sociales (MASHS 2009), Toulouse, Université Toulouse 2 Le Mirail.

Ratinaud Pierre, Marchand Pascal, 2012, "Application de la méthode Alceste à de "gros" corpus et stabilité des "mondes lexicaux". Analyse du "CableGate" avec Iramuteq ", Actes des JADT 2012, Liège, p. 835-844.

REESE Stephen, 2007, «The Framing Project. A bridging model for media research revisited », Journal of Communication, nº 57, p. 148-154.

REINERT Max, 1983, "Une méthode de classification descendante hiérarchique. Application à l'analyse lexicale par contexte», Les Cahiers de l'analyse des données, vol. VIII, nº 2, p. 187-198.

- 2008, «Mondes lexicaux stabilisés et analyse statistique de discours », JADT 2008, Lyon.

Ringoot Roselyne, RochARD Yvon, 2005, «Proximité éditoriale. Normes et usages des genres journalistiques », Mots. Les langages du politique, $\mathrm{n}^{\circ} 77$, Proximité, p.73-90. 
TANKARD James W., 2001, «The empirical approach to the study of media framing », Framing Public Life. Perspectives on Media and our Understanding of the Social World, S. Reese, O. Gandy, A.Grant éd., Mahwah (USA), Erlbaum, p. 95-106.

Touboul Annelise, Damian-Gaillard Béatrice, MARTy Emmanuel, 2012, "La disparité des modes de traitement journalistiques et des énonciations éditoriales sur le web. Le cas d'un sondage sur Marine Le Pen et la présidentielle de 2012 », Réseaux, $n^{0} 176$, p. 75-106. 